\title{
Nebulized nitrite protects rat lung grafts from ischemia reperfusion injury
}

\author{
Toshihiro Okamoto, MD, PhD, ${ }^{\mathrm{a}}$ Xiaoying Tang, MS, ${ }^{\mathrm{a}}$ Allison Janocha, BS, ${ }^{\mathrm{a}}$ Caral F. Farver, MD, ${ }^{\mathrm{c}}$ \\ Mark T. Gladwin, MD, ${ }^{\mathrm{d}}$ and Kenneth R. McCurry, MD ${ }^{\mathrm{a}, \mathrm{b}}$
}

Objectives: Nebulization is a potential method for delivering therapeutic agents to lung grafts. Recent evidence suggests that nitrite may mitigate ischemia-reperfusion injury via a nitric oxide-dependent pathway.

\begin{abstract}
Methods: Syngeneic orthotopic left lung transplantation was performed in rats after 7 hours of cold ischemia. Sodium nitrite $(3 \mathrm{mg}$ ) or phosphate-buffered saline (controls) was delivered before procurement via nebulization.
\end{abstract}

\begin{abstract}
Results: Nitrite treatment was associated with better oxygenation, lower peak airway pressure, lower wet/dry ratio, reduced myeloperoxidase level and macrophage infiltration, increased cyclic guanosine monophosphate (cGMP) levels, and decreased levels of interleukin 6 , interleukin 1- $\beta$, inducible nitric oxide synthase, and intercellular adhesion molecule-1 at 2 hours after reperfusion. Treatment with 2-(4-carboxypheny)4,4,5,5-tetramethylimidazoline-1-oxyl-3-oxide, a nitric oxide scavenger, reversed the beneficial effects of nitrite and decreased cGMP concentration in grafts. A dose-response curve of nitrite was performed at the following doses: $0.3 \mathrm{mg}$ (N0.1), $3.0 \mathrm{mg}$ (N1.0), $5.25 \mathrm{mg}$ (N1.75), $7.5 \mathrm{mg}$ (N2.5), and $15.0 \mathrm{mg}$ (N5.0). All treatments, excluding N1.0, resulted in poorer oxygenation, higher peak airway pressures, and higher wet/dry ratio. Higher dosage groups (N1.75, N2.5, and N5.0) exhibited positive immunostaining of nitrotyrosine and increased the intensity of nitrotyrosine in immunoblotting.
\end{abstract}

Conclusions: These data suggest that nebulized nitrite limits lung ischemia-reperfusion injury and may prove a clinically useful strategy but requires appropriate dosing to limit oxidative injury at high doses. (J Thorac Cardiovasc Surg 2013;145:1108-16)

Supplemental material is available online.

The lung is particularly susceptible to ischemia-reperfusion injury (IRI), which is in part why lung transplantation (LTx) remains clinically challenging. Severe IRI occurs in up to $25 \%$ of LTx recipients, leading to severe acute graft dysfunction and significantly increasing the 30-day mortality risk. ${ }^{1}$ IRI also causes enhanced cellular and humoral immune responses against the graft, elevating the risk of both acute and chronic rejection and resulting in diminished long-term recipient survival. ${ }^{2}$ The pathophysiologic events involved in lung IRI include oxidative stress, a dysregulated

From the Department of Pathobiology, ${ }^{\text {a }}$ Lerner Research Institute, and the Departments of Thoracic and Cardiovascular Surgery ${ }^{\mathrm{b}}$ and Anatomic Pathology, ${ }^{\mathrm{c}}$ Cleveland Clinic, Cleveland, Ohio; and the Division of Pulmonary, ${ }^{\mathrm{d}}$ Allergy and Critical Care Medicine and the Vascular Medicine Institute, University of Pittsburgh School of Medicine, Pittsburgh, Pa.

Disclosures: Authors have nothing to disclose with regard to commercial support.

Received for publication Jan 22, 2012; revisions received March 14, 2012; accepted

for publication April 5, 2012; available ahead of print Nov 12, 2012.

Address for reprints: Kenneth R. McCurry, MD, 9500 Euclid Ave, Cleveland, Ohio

44195 (E-mail: mccurrk@ccf.org or okamott@ccf.org).

$0022-5223 / \$ 36.00$

Copyright $\Subset 2013$ Published by Elsevier Inc. on behalf of The American Association for Thoracic Surgery

doi:10.1016/j.jtcvs.2012.04.006 inflammatory response, enhanced vascular thrombosis, and apoptosis. ${ }^{3}$ Diminishing these events could have significant beneficial effects, improving the outcome of LTx recipients. Moreover, effective strategies to reduce lung IRI could potentially expand the use of marginal donor organs, which are particularly susceptible to IRI, as well as facilitate further expansion of the donor pool to donation after cardiac death (DCD) lungs, which are subject to an obligate period of warm ischemia.

Nitric oxide (NO) has been demonstrated to mitigate IRI in many models and is a potential therapeutic agent for preventing lung IRI. However, some studies examining the inhalational delivery of $\mathrm{NO}$ in experimental and clinical LTx have shown benefit, ${ }^{4,5}$ whereas others have shown detrimental or neutral effects. ${ }^{6}$ These studies suggest that NO can be either protective or toxic to lung grafts depending on dose, timing, and duration of exposure and that there is a narrow therapeutic window for NO. ${ }^{7}$ Previously, nitrite was considered a stable product of NO metabolism in vivo. However, evidence now suggests that nitrite is an important reservoir of $\mathrm{NO}$ and that the reduction of nitrite to $\mathrm{NO}$ and other NO-containing molecules results in vasodilatation and blood flow regulation, especially under acidotic and hypoxic conditions. ${ }^{8}$ Several studies have also recently shown that nitrite possesses potent cytoprotective properties, including reduced apoptosis and cytotoxicity, in the setting 


$$
\begin{aligned}
& \text { Abbreviations and Acronyms } \\
& \text { cGMP = cyclic guanosine monophosphate } \\
& \text { C-PTIO = 2-(4-carboxypheny)-4,4,5,5- } \\
& \text { tetramethylimidazoline-1-oxyl-3-oxide } \\
& \text { DCD = donation after cardiac death } \\
& \text { ELISA = enzyme-linked immunosorbent assay } \\
& \text { IL = interleukin } \\
& \text { IRI = ischemia-reperfusion injury } \\
& \text { LTx = lung transplantation } \\
& \text { MPO = myeloperoxidase } \\
& \mathrm{NO}=\text { nitric oxide } \\
& \text { PBS = phosphate-buffered saline solution } \\
& \text { PAWP = peak airway pressure } \\
& \mathrm{PO}_{2}=\text { oxygen tension } \\
& \text { RT-PCR }=\text { reverse-transcriptase polymerase chain }
\end{aligned}
$$

of IRI. ${ }^{9,10}$ Moreover, recent studies have also revealed that nitrite contributes to hypoxic vasodilation in the lung and that nitrite inhibits hypoxic and inflammation-associated pulmonary arterial hypertension. ${ }^{11,12}$ Although the mechanism of its cytoprotective effects against IRI remain unclear, multiple studies support the idea that nitrite is reduced to NO with subsequent cytoprotection via mechanisms paralleling those hypothesized for NOmediated cytoprotection. ${ }^{7,13}$

We have previously demonstrated that nitrite can significantly inhibit IRI via an NO-dependent pathway when administered intravenously before the onset of cold ischemia. ${ }^{14}$ Translation of IRI therapeutic strategies to the clinic in transplantation have been hampered by delivery systems that require systemic administration in the setting of a multiorgan donor. Because the lung is unique in that therapeutic agents can be delivered via inhalation, we aimed to evaluate and optimize a clinically applicable strategy using nebulized nitrite to mitigate lung IRI. An inhalational delivery strategy could also potentially be applied in the setting of DCD donors or in the setting of ex vivo perfusion.

\section{MATERIAL AND METHODS \\ Animals}

The study protocol was approved by the Cleveland Clinic's Institutional Animal Care and Use Committee and all animals received humane care in compliance with the "Guide for the Humane Care and Use of Laboratory Animals" (Institute of Laboratory Animal Resources, National Research Council, National Academy Press, Washington, DC, 1996). Specific pathogen-free inbred male LEW (RT1 $1^{1}$ ) rats (Harlan Sprague Dawley Inc, Indianapolis, Ind) weighing 290 to $350 \mathrm{~g}$ were used.

\section{Orthotopic Left LTx in Rats}

Syngeneic orthotopic left LTx was performed using a cuff technique as previously described. ${ }^{15}$ In brief, donors received $250 \mu \mathrm{L}$ of phosphate- buffered saline solution (PBS) or $3.0 \mathrm{mg}$ of sodium nitrite (Sigma-Aldrich, St Louis, Mo) in $250 \mu \mathrm{L}$ of PBS via nebulization using the MicroSprayer model IA-1C (Penn-Century Inc, Philadelphia, Pa) after induction of anesthesia. Donor rats then underwent tracheotomy and mechanical ventilation at a positive end-expiratory pressure of $2.0 \mathrm{~cm}$ with a mixture of $21 \% \mathrm{ox}-$ ygen and isoflurane (Hospira Inc, Lake Forest, Ill). The lungs were flushed through the main pulmonary artery with $20 \mathrm{~mL}$ of $4^{\circ} \mathrm{C}$ Perfadex (Vitrolife, Göteborg, Sweden) 30 minutes after nebulization and excised. Grafts were implanted after 7 hours of cold storage at $4^{\circ} \mathrm{C}$ in Perfadex. Recipient rats were then extubated and given postoperative care.

\section{Experimental Groups}

Rats were randomly allocated into 3 groups: sham, control, and nitrite ( $n=7$ in each). In the sham group, lungs were harvested under anesthesia, frozen in liquid nitrogen, and stored at $-80^{\circ} \mathrm{C}$. In the control group, after PBS nebulization during harvest, the left lungs were preserved for 7 hours at $4^{\circ} \mathrm{C}$ and transplanted. In the nitrite group, after nebulization with $3.0 \mathrm{mg}$ of sodium nitrite dissolved in $250 \mu \mathrm{L}$ of PBS, the left lungs were preserved for 7 hours at $4^{\circ} \mathrm{C}$ and transplanted.

\section{Nebulized Nitrite Dose Response}

After initial experiments with nebulization of $3.0 \mathrm{mg}$ of sodium nitrite (N1.0), a dose response was performed. LTx were performed with the following doses of sodium nitrite: $0.3 \mathrm{mg}$ (N0.1, which means that 0.1 of the standard dose was given), $5.25 \mathrm{mg}$ (N1.75), $7.5 \mathrm{mg}(\mathrm{N} 2.5)$, and $15.0 \mathrm{mg}$ (N5.0) ( $\mathrm{n}=5$ in each group).

\section{Assessing the Effect of 2-(4-Carboxypheny)-4,4,5,5- Tetramethylimidazoline-1-Oxyl-3-Oxide (C-PTIO), an NO Scavenger}

Two additional groups were administered $3.1 \mathrm{mg} / \mathrm{kg}$ intravenously of C-PTIO (ENZO Life Sciences, Plymouth Meeting, Pa), an NO scavenger, at different time points after nebulization of $3.0 \mathrm{mg}$ of sodium nitrite to donors. In the C-PTIO 1 group $(\mathrm{n}=5)$, C-PTIO was given to the donors 10 minutes before cold ischemia during harvest, whereas in the C-PTIO 2 group $(n=5), C-P T I O$ was intravenously injected in the recipients 10 minutes before reperfusion.

\section{Postoperative Physiologic Evaluation and Tissue Preparation}

Following 2 hours of reperfusion after transplantation, graft pulmonary vein oxygen tension $\left(\mathrm{PO}_{2}\right)$ was evaluated (i-STAT, Abbott Point of Care, East Windsor, NJ) after a recruitment maneuver under anesthesia (inspired oxygen fraction $=1.0$ ). The blood sample of graft pulmonary vein was obtained exactly from the pulmonary vein cuff using a 25 -gauge needle under microscope guidance. The peak airway pressure (PAWP) of the trachea was recorded. Blood was obtained and immediately centrifuged at $7000 \mathrm{rpm}$ for 6 minutes and the plasma was frozen in liquid nitrogen and stored at $-80^{\circ} \mathrm{C}$. Graft tissue was retrieved after flushing of the pulmonary artery with PBS, frozen in liquid nitrogen, stored at $-80^{\circ} \mathrm{C}$, and used for reversetranscriptase polymerase chain reaction (RT-PCR), enzyme-linked immunosorbent assay (ELISA), and Western blotting analysis.

\section{Methemoglobin Levels and Nitrite Concentrations}

Blood samples were obtained from the abdominal aorta 30 minutes after sodium nitrite nebulization in the donor, and methemoglobin levels were immediately measured using an ABL 725 (Radiometer Ag, Copenhagen, Denmark). The right lung was flushed by air-equilibrated PBS supplemented with $N$-ethylmaleimide/ethylenediaminetetraacetic acid $(10 / 2.5 \mathrm{mmol} / \mathrm{L})$ (NEM; Sigma-Aldrich) in the donor 30 minutes after nebulization and stored at $-80^{\circ} \mathrm{C}$. After lung tissue was homogenized, nitrite concentration in tissue lysates was measured using NOA 280i (GE, 
Boulder, Colo). The plasma nitrite concentration was also measured using the same machine.

\section{Wet/Dry Ratio}

The wet weight of frozen lung samples was measured and lungs were then dried in an oven at $60^{\circ} \mathrm{C}$ for 1 day to obtain the dry weight.

\section{Real-Time RT-PCR}

RNA was extracted from frozen lung tissue by using TRIzol (Invitrogen, Carlsbad, Calif). The mRNA for interleukin (IL) 6 , IL-1 $\beta$, tumor necrosis factor-alpha, inducible NO synthase, IL-10, intercellular adhesion molecule-1, and glyceraldehyde 3-phosphate dehydrogenase were quantified with iQ SYBR Green Supermix (BIO-RAD, Hercules, Calif), as previously reported. ${ }^{16}$ The expression of each gene was normalized with glyceraldehyde 3-phosphate dehydrogenase.

\section{Myeloperoxidase Activity}

Myeloperoxidase (MPO) concentration was evaluated using the Rat MPO ELISA kit (HyCult Biotechnology, Uden, The Netherlands).

\section{Cyclic Guanosine Monophosphate Measurement \\ Cyclic guanosine monophosphate (cGMP) was analyzed in lung tissue with the Cyclic GMP EIA kit (Cayman Chemical Company, Ann Arbor, Mich). Results were expressed in picomoles ( $\mathrm{pmol}$ ) of cGMP per milligram of protein.}

\section{Histopathologic Analysis}

Formalin-fixed, paraffin-embedded graft tissue was sectioned at $5 \mu \mathrm{m}$. Sections were incubated with monoclonal mouse anti-rat ED1 antibody (Serotec, Raleigh, NC) and anti-nitrotyrosine antibody (Millipore, Temecula, Calif) followed by LSAB horseradish peroxidase detection (DAKO, Carpenteria, Calif). For ED1 evaluation, the ratio of positive staining to negative staining was calculated.

\section{Western Blot Analysis}

Tissues were homogenized in lysis buffer, electrophoresed on NuPAGE $4 \%-12 \%$ Bis-Tris gel (Invitrogen), and transferred to nitrocellulose membranes (Invitrogen). Membranes were washed and then incubated with mouse antibodies for nitrotyrosine (Millipore, Temecula, Calif) and rabbit polyclonal actin (Sigma-Aldrich) followed by incubation with secondary antibody for 2 hours. Membranes were detected using Immobilon Western HRP Substrate Peroxide Solution (Millipore, Billerica, Mass) and the Kodak 4000R Image Station (Kodak, Rochester, NY).

\section{Statistical Analysis}

All values are expressed as mean \pm standard error of the mean. Data were evaluated by analysis of variance or the Student $t$ test. Post hoc test for the analysis of variance included the Bonferroni multiple comparison test. All analyses were performed on SigmaStat software version 3.5 (Systat Software Inc, Richmond, Calif). A value of $P<.05$ was used to distinguish the differences by chance.

\section{RESULTS}

\section{Nebulized Nitrite Improved Lung Graft Function}

The nitrite group ( $3.0 \mathrm{mg}$ sodium nitrite nebulization) had higher $\mathrm{PO}_{2}$ levels than the control group (PBS nebulization) $(560.8 \pm 29.0$ vs $164.0 \pm 38.2 \mathrm{~mm} \mathrm{Hg} ; P<.001$; inspired oxygen fraction $=1.0$ ). The level of $\mathrm{Po}_{2}$ in the nitrite group was equivalent to that in the sham group $(612.5 \pm 15.5 \mathrm{~mm}$ $\mathrm{Hg})$. The nitrite group had lower PAWP than the control group $(5.8 \pm 0.3$ vs $7.1 \pm 0.4 \mathrm{~mm} \mathrm{Hg} ; P=.04)$ and the nitrite group also had a lower wet/dry ratio than the control group (7.23 \pm 0.58 vs $8.05 \pm 0.34 ; P=.66$ ) (Figure $1, A)$.

\section{Dose-Dependent Cytoprotective Effects of Nebulized Nitrite in Lung IRI}

To determine the optimal dosage of nebulized nitrite, we assessed lung graft physiologic function after 2 hours of reperfusion at various dosages of nitrite. In the N0.1 group (0.3 mg sodium nitrite), $\mathrm{PO}_{2}$ was reduced compared with that in the N1.0 group $(3.0 \mathrm{mg})(P<.001)$. In the higher dosage groups $(5.25 \mathrm{mg}, 7.5 \mathrm{mg}$, and $15.0 \mathrm{mg}), \mathrm{Po}_{2}$ levels were lower than that in the N1.0 group $(P<.01)$. Both lower dosage (N0.1) and higher dosage groups (N1.75, N2.5, and N5.0) had higher PAWP levels than the N1.0 group. The wet/dry ratio in the N1.0 group was lower than those in the higher dosage groups (Figure 1,B).

\section{Methemoglobin Levels in the Blood, Lung Tissue Nitrite Concentration, and Plasma Nitrite Concentration 30 Minutes After Nebulization in Donor Animals}

There was a dose-dependent increase in methemoglobin levels. The methemoglobin level for the N5.0 group was above the upper limit level of $37 \%$ (Figure 2, A). The nitrite level in the tissue and the plasma also increased in a dosedependent manner (Figure 2, $B$ and $C$ ). The N1.0 group had $6.2 \% \pm 0.2 \%$ methemoglobin, $80.0 \pm 10.5 \mathrm{pmol} / \mathrm{mg}$ of tissue nitrite, and $199.3 \pm 23.2 \mu \mathrm{mol} / \mathrm{L}$ of plasma nitrite after nebulization.

\section{Lung Tissue and Plasma Nitrite Concentrations After 2 Hours of Reperfusion in Recipient Animals}

To obtain a pharmacokinetic profile for nebulized nitrite, we measured the plasma nitrite concentration 2 hours after reperfusion in the control and various dosage groups. The plasma nitrite mean concentrations of all groups were within the natural range of $0.1 \mu \mathrm{mol} / \mathrm{L}$ to $0.8 \mu \mathrm{mol} / \mathrm{L}$ (Figure 2, D). ${ }^{17}$ The tissue nitrite concentration in the N1.0 group after 2 hours of reperfusion was reduced to $1.3 \pm 0.5 \mathrm{pmol} / \mathrm{mg}$, which was similar to that in the sham group 30 minutes after nebulization $(0.9 \pm 0.0 \mathrm{pmol} / \mathrm{mg})$ (Figure 2,B).

\section{Excessive Formation of Peroxynitrite in Higher Dosage Groups}

To evaluate peroxynitrite formation as a marker of sodium nitrite toxicity, we performed immunostaining with anti-3-nitrotyrosine in the graft lungs. In the N1.0 and N0.1 groups, no immunostaining for nitrotyrosine was observed. In the higher dosage groups (N1.75, N2.5, and N5.0), epithelial and smooth muscle cells were positive in all bronchi and bronchioles and all smooth muscle cells within the vessel walls were positive (Figure 3,A). 

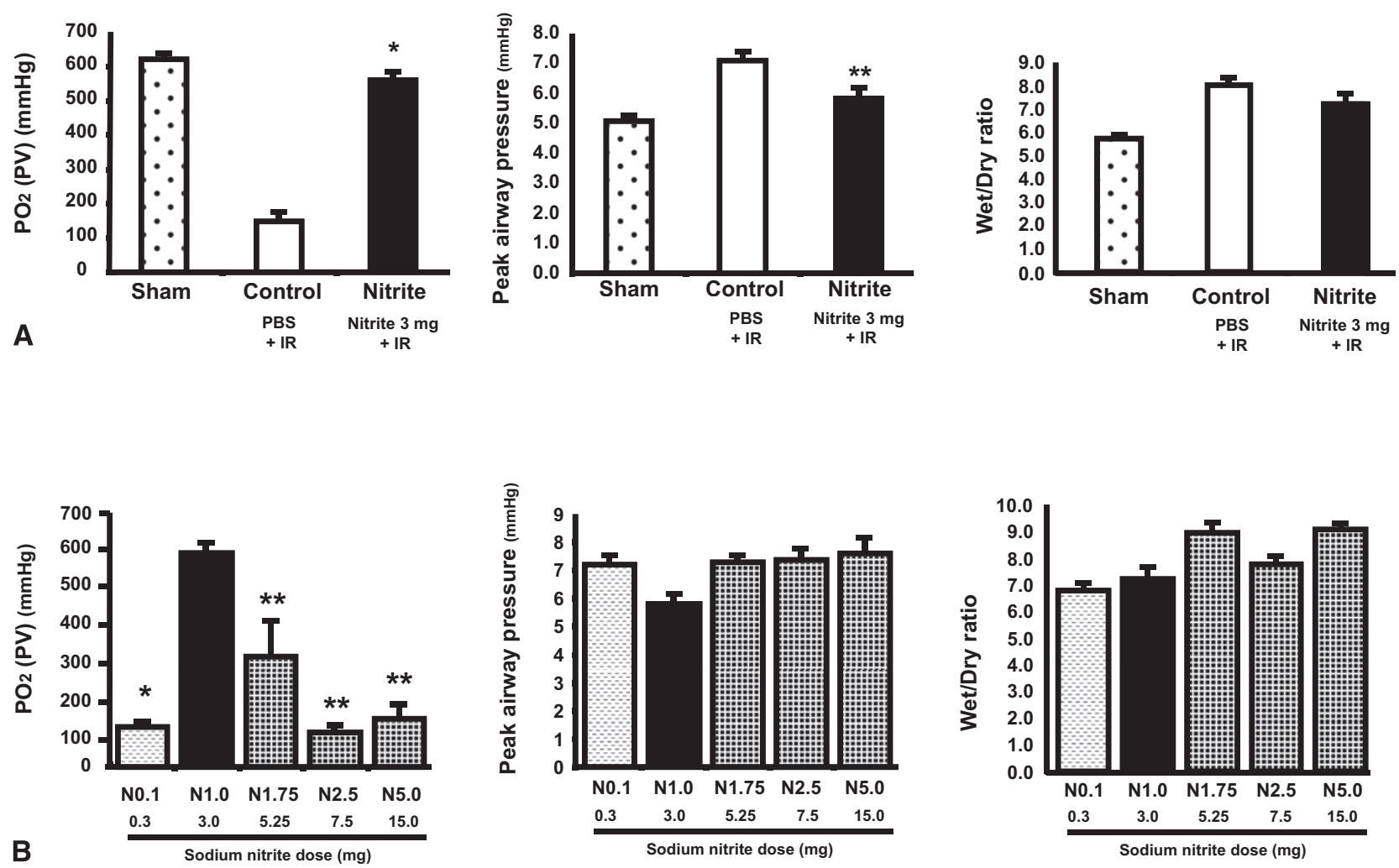

FIGURE 1. Lung graft physiologic function in experimental groups. A, Pulmonary vein oxygen tension $\left(\mathrm{Po}_{2}\right)$, peak airway pressure $(P A W P)$ of the trachea, and graft wet/dry ratio after 2 hours of reperfusion (inspired oxygen fraction $=1.0$ ) in sham, control, and nitrite groups. The nitrite nebulization group (nitrite, $\mathrm{n}=7)$ had higher $\mathrm{Po}_{2}$ levels than the phosphate-buffered saline $(P B S)$ nebulization group (control, $\left.\mathrm{n}=7\right)(560.8 \pm 29.0 \mathrm{~mm} \mathrm{Hg} \mathrm{vs} 164.0 \pm 38.2 \mathrm{~mm} \mathrm{Hg}$; $* P<.001)$. The nitrite group had lower PAWP than the control group $(5.8 \pm 0.3 \mathrm{~mm} \mathrm{Hg}$ vs $7.1 \pm 0.4 \mathrm{~mm} \mathrm{Hg} ; * P=.04)$. The graft wet/dry ratio in the nitrite group was lower than that in the control group $(7.23 \pm 0.58 \mathrm{vs} 8.05 \pm 0.34 ; P=.66)$. B, Lung graft physiologic function in various dosage groups $(0.3 \mathrm{mg}$ sodium nitrite in $\mathrm{N} 0.1,5.25 \mathrm{mg}$ in N1.75, 7.5 mg in N2.5, and $15.0 \mathrm{mg}$ in N5.0; $\mathrm{n}=5$ each) in addition to the standard nitrite group ( $\mathrm{N} 1.0,3.0 \mathrm{mg}$ ). In the N0.1 group, Po 2 was reduced compared with that in the nitrite group (N1.0) $\left(103.6 \pm 18.4 \mathrm{~mm} \mathrm{Hg}\right.$ vs $\left.560.8 \pm 29.0 \mathrm{~mm} \mathrm{Hg} ;{ }^{*} P<.001\right)$. In higher dosage groups (N1.75, $\mathrm{N} 2.5$, and $\mathrm{N} 5.0), \mathrm{Po}_{2}$ levels were lower than those in the nitrite group (N1.0) $(289.8 \pm 104.1 \mathrm{~mm} \mathrm{Hg}, 90.4 \pm 20.5 \mathrm{~mm} \mathrm{Hg}, 127.0 \pm 49.1 \mathrm{~mm} \mathrm{Hg}$; ** $P<.01)$. The N0.1 group had higher levels of PAWP than the nitrite group (N1.0) $(7.2 \pm 0.4 \mathrm{~mm} \mathrm{Hg}$ vs $5.8 \pm 0.3 \mathrm{~mm} \mathrm{Hg})$. All higher dosage groups (N1.75, N2.5, and N5.0) had higher levels of PAWP than the nitrite group $(7.3 \pm 0.2 \mathrm{~mm} \mathrm{Hg}, 7.3 \pm 0.4 \mathrm{~mm} \mathrm{Hg}$, and $7.6 \pm 0.6 \mathrm{~mm} \mathrm{Hg}$, respectively; $P=.08)$. The graft wet/dry ratio in the N1.0 group was lower than those in higher dosage groups (N1.75, N2.5, and N5.0). PV, Pulmonary vein; $I R$, ischemia reperfusion.

Moreover, we performed Western blotting to quantify nitrotyrosine protein adducts in the graft lungs after 2 hours of reperfusion in various dosage groups. Consistent with our immunostaining results, there were marked increases in nitrotyrosine immunoreactivity in the higher dosage groups compared with those in the N0.1 and N1.0 groups. The main nitrotyrosine band intensity compared with that of $\beta$-actin was increased in the higher dosage groups relative to that in the N0.1 and N1.0 groups (Figure 3, $B$ and $C$ ).

\section{Cytoprotective Effects of Optimal Dose of Nebulized Nitrite via Inhibition of Proinflammatory Mediators, MPO Activity, and Macrophage Infiltration}

In lung grafts exposed to $3.0 \mathrm{mg}$ of inhaled sodium nitrite, the expression of IL- 6 , IL- $1 \beta$, inducible NO synthase, and intercellular adhesion molecule- 1 in RT-PCR analysis were lower than that in the control group $(P<.05)$. The
IL-10 level in the nitrite group was lower than that in the control group $(P=.08)$ (Figure $4, A)$. Lung neutrophil infiltration is a hallmark of lung IRI. Using ELISA, we measured MPO activity in the graft, as a marker of neutrophil sequestration. The nitrite group had lower levels of MPO activity than the control group $(17.6 \pm 2.4$ vs $31.4 \pm 4.9$ $\mathrm{ng} / \mathrm{mL} ; P=.02$ ) (Figure $4, B$ ). Immunohistochemical staining for activated macrophages (ED1+) revealed that nitrite nebulization reduced the ratio of ED1-positive cells $(9.7 \% \pm 0.3 \%$ vs $18.3 \% \pm 1.0 \% ; P<.001$ ) (Figure $4, C$ ).

\section{Elevation of cGMP Levels in the Graft Lung Induced by Nebulized Nitrite}

In other models of ischemic injury, evidence suggests that the cytoprotective effect of nitrite is mediated by conversion of nitrite to NO in ischemic tissue beds. ${ }^{18}$ Inasmuch as there is considerable evidence demonstrating that the primary signaling pathway for $\mathrm{NO}$ is through activation of soluble 

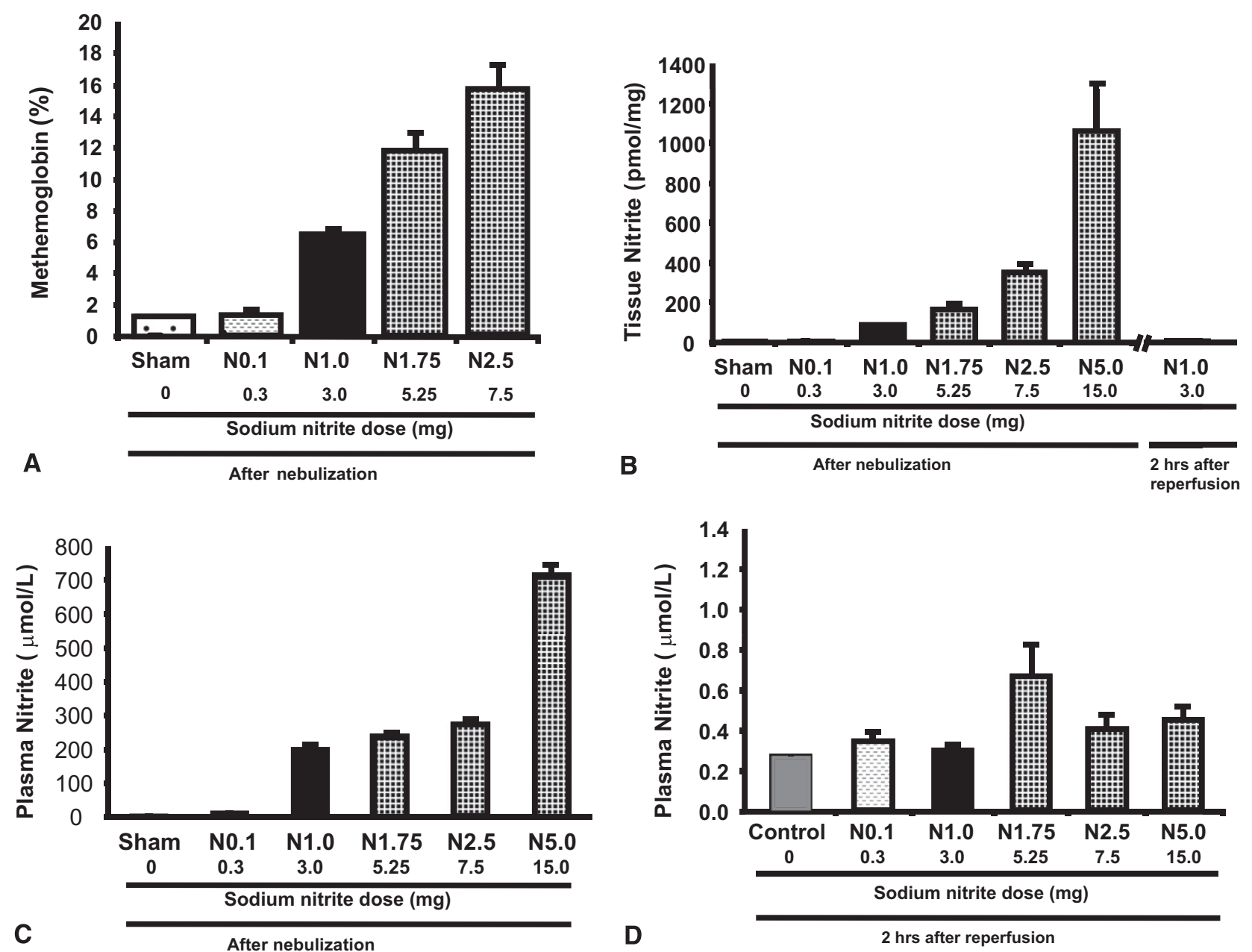

FIGURE 2. Methemoglobin levels and nitrite concentrations in lung tissue and plasma. A, The methemoglobin level of the nitrite group (N1.0) was 6.2\% $\pm 0.2 \%$ in donor rats 30 minutes after nebulization. The methemoglobin level increased in a dose-dependent manner. $(1.1 \% \pm 0.0 \%$ in sham, $1.3 \% \pm 0.0 \%$ in N0.1 $11.7 \% \pm 1.1 \%$ in N1.75, and $15.6 \% \pm 1.5 \%$ in N2.5). Data for the N5.0 group were above the upper limit level of 37\%. B, The tissue nitrite concentration in the N1.0 group was $80.0 \pm 10.5 \mathrm{pmol} / \mathrm{mg}$ in donor lungs 30 minutes after nebulization, while that in N0.1 was $6.6 \pm 1.9 \mathrm{pmol} / \mathrm{mg}$. There was a dose-dependent increase in the lung tissue nitrite concentration in donor lungs at all dosages. The tissue nitrite concentration of recipient lungs in the $\mathrm{N} 1.0$ group was $1.3 \pm 0.5 \mathrm{pmol} / \mathrm{mg}$ after 2 hours of reperfusion. C, The plasma nitrite concentration was measured in grafts 30 minutes after nebulization. The nitrite level of N0.1 and N1.0 was $10.0 \pm 0.6 \mu \mathrm{mol} / \mathrm{L}$ and $199.3 \pm 23.2 \mu \mathrm{mol} / \mathrm{L}$, respectively. D, The plasma nitrite concentration was measured in recipient rats after 2 hours of reperfusion. The plasma nitrite concentration in the $\mathrm{N} 1.0$ group was $0.34 \pm 0.04 \mu \mathrm{mol} / \mathrm{L}$. The means in all groups were within the normal physiologic range of 0.1 to $0.8 \mu \mathrm{mol} / \mathrm{L}$.

guanylyl cyclase resulting in the formation of cGMP, we measured cGMP levels in lung graft tissue 2 hours after reperfusion. There was an increase in the cGMP concentration in the nitrite group compared with that in the control group $(P=.04)$. The N1.75, N2.5, and N5.0 groups demonstrated even higher cGMP concentrations than the nitrite (N1.0) group (Figure 5).

\section{Reversal of the Cytoprotective Effects of C-PTIO by Nitrite}

To further investigate the mechanism of the cytoprotective effect of nitrite in our model, we examined whether the tissue protective effect of nebulized nitrite $(3.0 \mathrm{mg})$ would be inhibited by C-PTIO, an NO scavenger. Our group has previously shown that C-PTIO treatment in donor rats induces no decline in oxygenation. ${ }^{14}$ In the CPTIO1 group, where C-PTIO was administered in donor animals before cold ischemia, there was a decrease in $\mathrm{Po}_{2}$ compared with that in the nitrite group $(80.2 \pm 20.3$ vs $560.8 \pm 26.8 \mathrm{~mm}$ $\mathrm{Hg} ; P<.001)$. In the CPTIO2 group, where C-PTIO was administered to recipient animals 10 minutes before reperfusion, there was also a decrease in $\mathrm{Po}_{2}$ compared with that in the nitrite group $(70.8 \pm 12.4$ vs $560.8 \pm 26.8 \mathrm{~mm} \mathrm{Hg}$; $P<.001$ ) (Figure E1, $A$ ). The CPTIO1 and CPTIO2 groups had higher values of PAWP $(P<.01$, each $)$ and higher wet/ dry ratios than the nitrite group (Figure E1, $B$ and $C$ ). The 

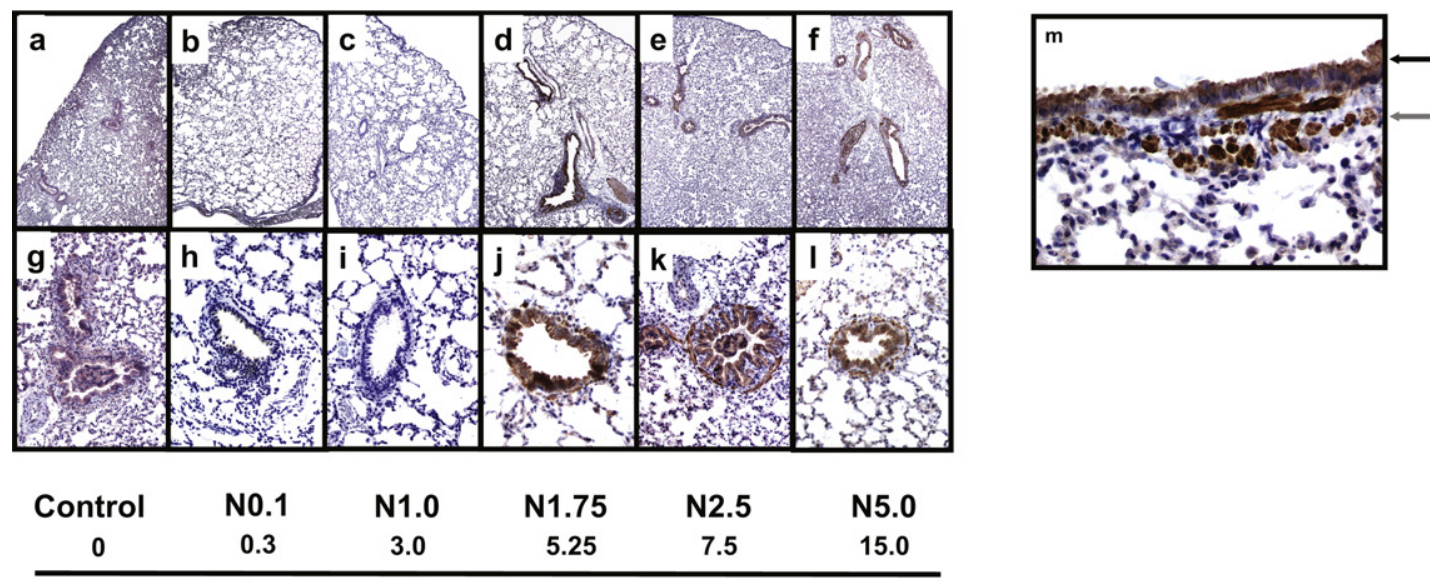

A

Sodium nitrite dose $(\mathrm{mg})$

Sodium nitrite dose $(\mathrm{mg})$
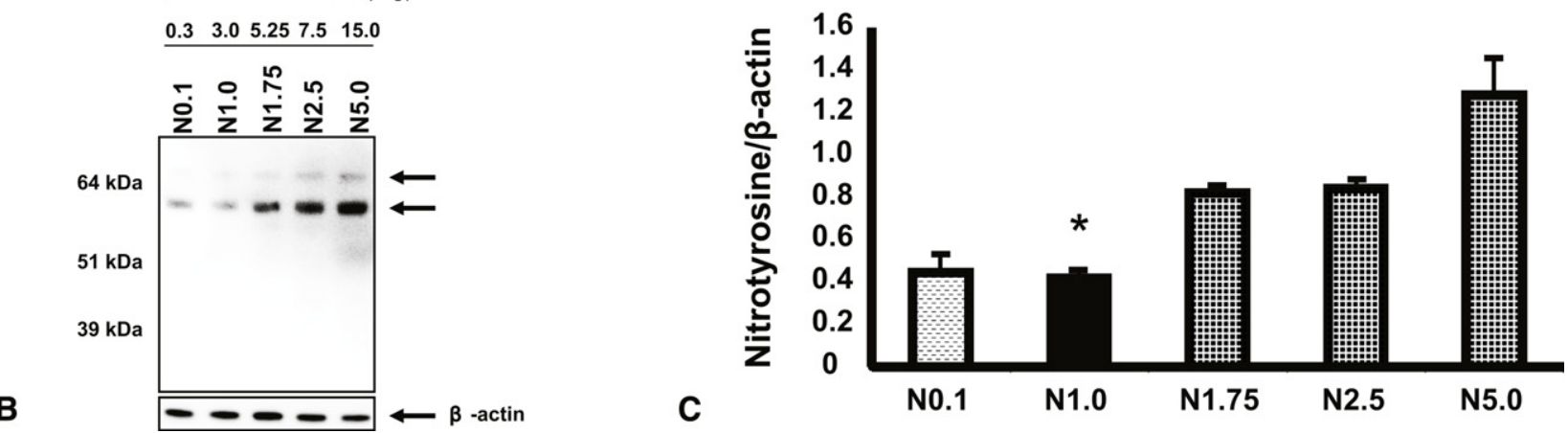

FIGURE 3. Nitrotyrosine immunostaining and Western blotting. A, In the N0.1 and N1.0 groups, nitrotyrosine staining was negative (b-c: hematoxylin, $\times 40$; h-i: hematoxylin, $\times 200$ ). In higher dosage groups (N1.75, N2.5, and N5.0), all bronchiole epithelial and smooth muscle cells were positively stained (d-f: hematoxylin, $\times 40$; j-1: hematoxylin, $\times 200$; k: hematoxylin, $\times 400$ ). In the control group, bronchial epithelial and smooth muscle cells were slightly positive (a: hematoxylin, $\times 40$; g: hematoxylin, $\times 200$ ). Arrows indicate positively stained epithelial cells (black) and smooth muscle cells $($ gray). B, Western blot analysis of nitrotyrosine in the tissue lysates of graft lungs after 2 hours of reperfusion in various dosage groups revealed marked increases in nitration in the higher dosage groups (N1.75, N2.5, and N5.0) relative to the levels in the N0.1 and N1.0 groups. C, Densitometric analysis revealed that the total nitrotyrosine band intensity compared with $\beta$-actin was increased in the higher dosage groups (N1.75, N2.5, and N5.0) relative to the levels in the N0.1 and N1.0 groups (N1.0 vs N5.0; *P=.009).

CPTIO1 and CPTIO2 groups had lower levels of cGMP than the nitrite group (Figure 5).

\section{DISCUSSION}

Previously, we have demonstrated that nitrite mitigates lung IRI via an NO-dependent pathway when delivered intravenously before the onset of cold ischemia in a lung transplant model. ${ }^{14}$ This work is consistent with other studies demonstrating that intravenous nitrite is cytoprotective in animal models of heart, liver, kidney, and brain IRI. ${ }^{10,19,20}$ In the present study, we demonstrate that delivery of nitrite to lung grafts via inhalation before the onset of ischemia mitigates IRI in a dose-dependent fashion and further demonstrate that high doses of inhaled nitrite are associated with toxicity and are less efficacious. Nebulization is theoretically an ideal method of delivery of therapeutic agents to the lungs. Whereas delivery of nitrite to lungs via nebulization has been reported in models of pulmonary hypertension and vascular injury, ${ }^{11-13}$ there have been no previously reported studies evaluating the efficacy of inhaled nitrite in the setting of LTx. The therapeutic efficacy observed as well as the findings of a dosedependent increase in donor plasma and lung tissue nitrite concentration as well as the notable increase in methemoglobin level in donor blood suggests that nebulization is an efficient method of drug delivery to lung grafts. Inasmuch as IRI therapeutics in transplantation have been hampered by the need for systemic administration of agents to donors, the current findings may advance the potential for clinical translation.

An important finding in our study was that nebulized nitrite exhibited dose-dependent cytoprotective effects on lung IRI as well as dose-dependent toxicity. The standard nitrite group (N1.0) inhibited lung IRI, whereas both lower 

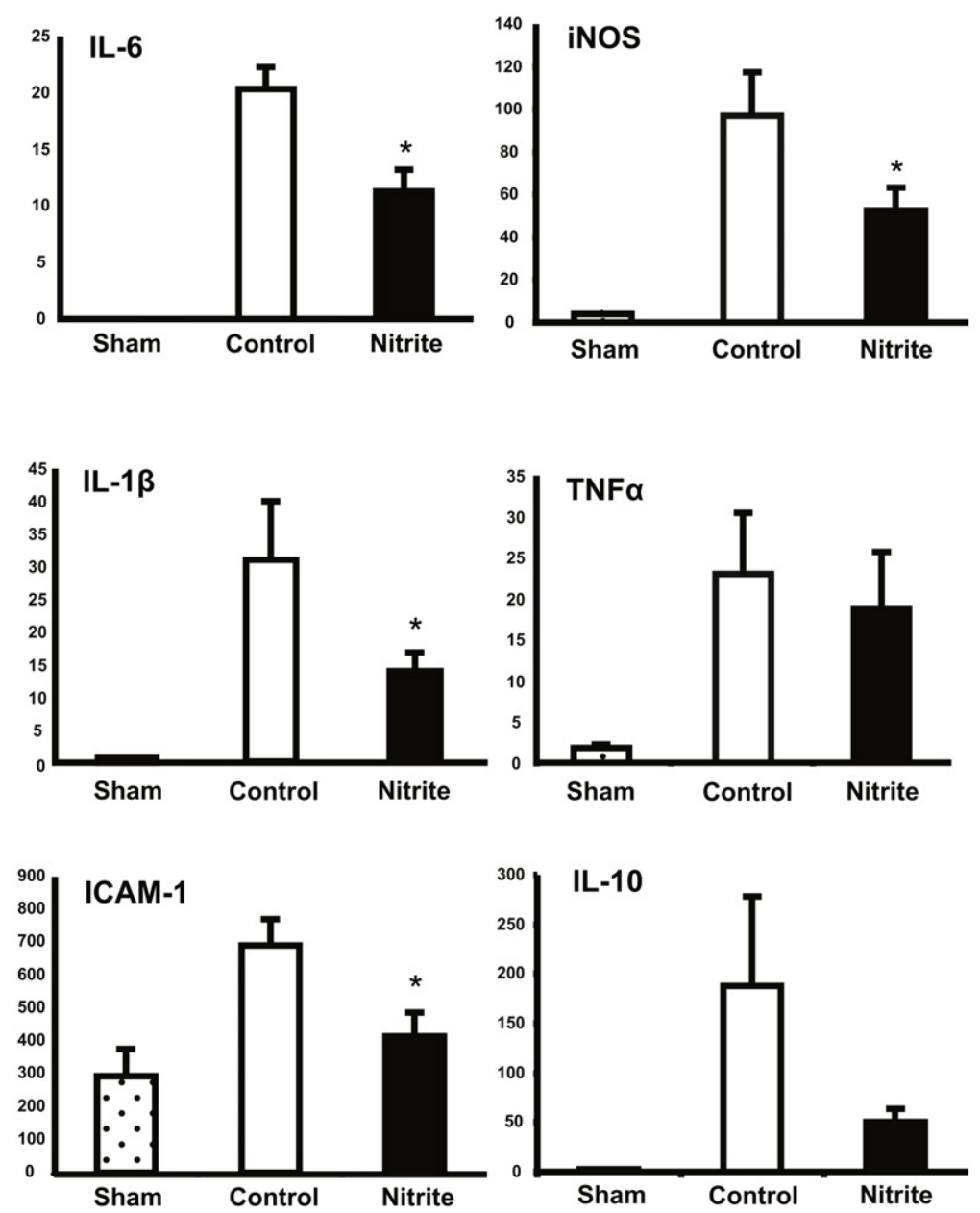

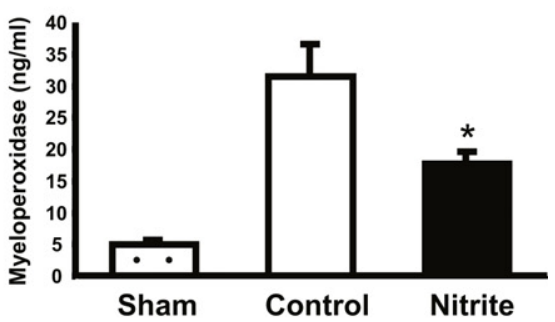

B
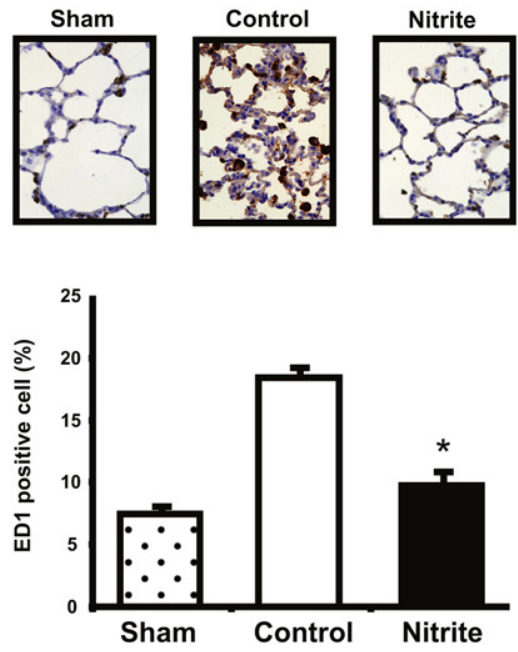

C

A

FIGURE 4. Proinflammatory mediators, myeloperoxidase activity, and macrophage infiltration. A, Reverse-transcriptase polymerase chain reaction (RT$P C R$ ) analysis of proinflammatory cytokines in rat graft lungs after 2 hours of reperfusion. RT-PCR analysis showed that the expression levels of interleukin (IL) 6, IL-1 $\beta$, inducible nitric oxide synthase (iNOS), and intercellular adhesion molecule-1 (ICAM-1) were lower in the nitrite group than in the control group $(* P<.05$, Student $t$ test). The IL-10 level in the nitrite group was lower than that in the control group $(P=.08)$. There was no difference in the tumor necrosis factor-alpha $(T N F \alpha)$ levels between the nitrite group and the control group $(P=.44)$. B, Myeloperoxidase activity in graft lung. The nitrite group exhibited lower levels of myeloperoxidase than the control group $(17.6 \pm 2.4 \mathrm{ng} / \mathrm{mL}$ vs $31.4 \pm 4.9 \mathrm{ng} / \mathrm{mL} ; * P=.02)$. C, Immunohistochemical staining of ED1 was observed in alveolar pneumocytes. The nitrite group had lower levels of ED1-positive cell than the control group $(9.7 \% \pm 0.3 \%$ vs $18.3 \% \pm 1.0 \%$; $* P<.001)$.

dosage (N0.1) and higher dosage (N1.75, N2.5, and N5.0) did not produce a protective effect on lung IRI. Toxicity was demonstrated by immunostaining and Western blotting analyses, which revealed increased levels of nitrated proteins only in the higher dosage groups $(\mathrm{N} 1.75, \mathrm{~N} 2.5$, and N5.0). This dose-dependent toxicity is congruent with reports on the toxic effects of excessive NO or nitrite levels, which lead to the intracellular generation of "reactive nitrogen species" such as peroxynitrite. ${ }^{18}$ These data are also consistent with Jung and associates,${ }^{10}$ who demonstrated that high-dose intravenous sodium nitrite had no cytoprotective effect in a mouse brain IRI model, whereas the optimal dose of nitrite prevented IRI. Duranski and associates ${ }^{19}$ also reported a dose-dependent tissue protective effect of intravenous nitrite in heart and liver IRI models. Our data suggest that nebulized nitrite also has a therapeutic range associated with efficacy and absence of toxicity and that $3.0 \mathrm{mg}$ of sodium nitrite could be a good therapeutic dose, being both efficacious and safe, for nebulization to donor lungs.

An important finding in our study was that the mechanism of effect of inhaled nitrite appears to be mediated via NO. Experiments using C-PTIO, a scavenger of NO, provide strong data suggesting that the cytoprotective effects of nitrite in our model are mediated via NO-dependent pathways after conversion of nitrite to NO, as shown in other reports. ${ }^{10,19,20}$ These data are further supported by the finding of a dose-dependent increase in lung tissue cGMP. 


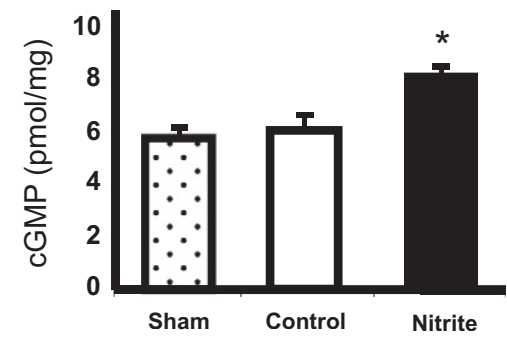

A

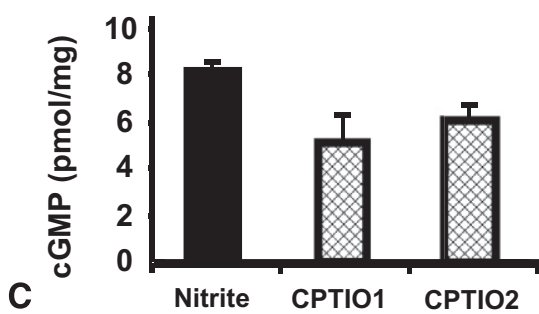

.

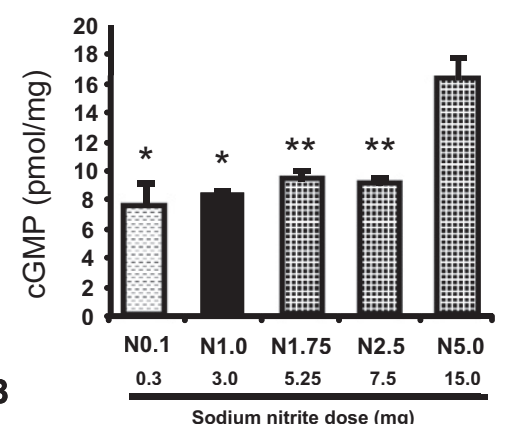

Sodium nitrite dose $(\mathrm{mg})$

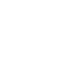

FIGURE 5. Cyclic guanosine monophosphate ( $c G M P$ ) level in lung graft. A, There was an increase in the cGMP concentration in the lung tissue after 2 hours of reperfusion in the nitrite group compared with that in the control group $(* P=.04)$. B, The N5.0 group had higher cGMP concentrations than the other dosage groups $\left({ }^{*} P<.001,{ }^{*} P<.01\right)$. C, The CPTIO1 and CPTIO2 groups had lower levels of cGMP than the nitrite group $(P=.051)$. C-PTIO, 2-(4-carboxypheny)-4,4,5,5-tetramethylimidazoline-1-oxyl-3-oxide.

Furthermore, the CPTIO1 group (C-PTIO delivered before onset of cold ischemia), which scavenged NO during ischemia and reperfusion, and the CPTIO2 group (C-PTIO delivered immediately before reperfusion), which scavenged $\mathrm{NO}$ only during reperfusion, demonstrated similar results, with significantly deteriorated pulmonary physiologic function in both groups. It is suggested from these data that the important period for nitrite/NO efficacy for the current delivery strategy was during reperfusion, not during cold storage, because the inhibitory action of C-PTIO in both the CPTIO1 and CPTIO2 groups overlapped only during reperfusion. Regarding NO therapeutics, there is controversy in the literature regarding the most beneficial timing of NO administration during ischemia or during reperfusion. Several studies support the administration of NO in early ischemia in lung ischemia models, ${ }^{21}$ wherreas other studies demonstrated more cytoprotective effects of NO during reperfusion than during ischemia in rat small bowel and lung ischemia models. ${ }^{22}$ Our present data using C-PTIO support the latter findings indirectly, although we did not conduct a direct evaluation using an additional group with the administration of nitrite during reperfusion.

In addition to evidence of cytoprotection by inhaled NO in rat lungs, ${ }^{23}$ much evidence suggests that several NO donors can inhibit lung IRI, including nitroglycerin and nitroprusside. ${ }^{24,25}$ As opposed to inhaled NO, a benefit of nitrite is that NO generation from nitrite would occur in tissue beds undergoing oxidative stress and at physiologic concentrations, thus minimizing the possibility of deleterious effects. Second, nitrite does not produce a tolerance phenomenon, which limits the therapeutic efficiency of nitroglycerin. ${ }^{24}$ The third benefit is that the measurement of nitrite concentrations in both blood and tissues can be easily performed to determine the optimal dosage and prevent toxicity, whereas NO is unstable and can be rapidly metabolized within a few seconds, making it difficult to determine the NO concentration in blood and tissue in clinical situations. Therefore, nebulized sodium nitrite may be safe and efficacious as a therapeutic agent in clinical LTx.

In this study, we have shown that nebulized nitrite mitigates lung IRI in a dose-dependent manner in a rat LTx model using standard, cold static lung storage. To expand the donor lung supply to meet demand, there has been much interest in the use of DCD lung donors as well as in ex vivo perfusion for lung resuscitation. Delivery of therapeutic agents to lungs on ex vivo perfusion circuits has been an area of interest of investigators and could potentially be applied in the setting of DCD donors (where the circulation has ceased) as well. Delivery of nitrite via inhalation in these scenarios could prove beneficial.

In conclusion, the present study demonstrates that nebulized nitrite has remarkable cytoprotective effects against IRI in a rat left LTx model. Although therapy using nebulized nitrite has a limited optimal dosage range, data from physiologic, immunologic, and molecular analyses, as well as pharmacokinetic evaluations, were consistent with the cytoprotective effect of sodium nitrite against lung IRI. The inhibition of NO by C-PTIO and the dosedependent generation of cGMP suggests that the effects of 
inhaled nitrite in this model are mediated by an NOdependent mechanism. This approach may hold promise for clinical translation.

We thank Linda Vargo for technical support in immunological analysis, Youran Fan for suggestions in statistic analysis, and Serpil Erzurum for helpful discussions.

\section{References}

1. Christie JD, Kotloff RM, Ahya VN, Tino G, Pochettino A, Gaughan C, et al. The effect of primary graft dysfunction on survival after lung transplantation. Am J Respir Crit Care Med. 2005;171:1312-6.

2. Bharat A, Kuo E, Steward N, Aloush A, Hachem R, Trulock E, et al. Immunological link between primary graft dysfunction and chronic lung allograft rejection. Ann Thorac Surg. 2008;86:189-95.

3. de Perrot M, Liu M, Waddell T, Keshavjee S. Ischemia-reperfusion-induced lung injury. Am J Respir Crit Care Med. 2003;167:490-511.

4. Pinsky D, Naka Y, Chowdhury N, Liao H, Oz M, Michler R, et al. The nitric oxide/cyclic GMP pathway in organ transplantation: critical role in successful lung preservation. Proc Natl Acad Sci U S A. 1994;91:12086-90.

5. Date H, Triantafillou A, Trulock E, Pohl M, Cooper J, Patterson G. Inhaled nitric oxide reduces human lung allograft dysfunction. J Thorac Cardiovasc Surg. 1996;111:913-9.

6. Meade M, Granton J, Matte-Martyn A, McRae K, Weaver B, Cripps P, et al. A randomized trial of inhaled nitric oxide to prevent ischemia-reperfusion injury after lung transplantation. Am J Respir Crit Care Med. 2003;167:1483-9.

7. Bolli R. Cardioprotective function of inducible nitric oxide synthase and role of nitric oxide in myocardial ischemia and preconditioning: an overview of a decade of research. J Mol Cell Cardiol. 2001;33:1897-918.

8. Webb A, Bond R, McLean P, Uppal R, Benjamin N, Ahluwalia A. Reduction of nitrite to nitric oxide during ischemia protects against myocardial ischemiareperfusion damage. Proc Natl Acad Sci U S A. 2004;101:13683-8.

9. Shiva S, Sack M, Greer J, Duranski M, Ringwood L, Burwell L, et al. Nitrite augments tolerance to ischemia/reperfusion injury via the modulation of mitochondrial electron transfer. J Exp Med. 2007;204:2089-102.

10. Jung K, Chu K, Ko S, Lee S, Sinn D, Park D, et al. Early intravenous infusion of sodium nitrite protects brain against in vivo ischemia-reperfusion injury. Stroke. 2006;37:2744-50.

11. Hunter C, Dejam A, Blood A, Shields H, Kim-Shapiro D, Machado R, et al. Inhaled nebulized nitrite is a hypoxia-sensitive NO-dependent selective pulmonary vasodilator. Nat Med. 2004;10:1122-7.
12. Zuckerbraun B, Shiva S, Ifedigbo E, Mathier M, Mollen K, Rao J, et al. Nitrite potently inhibits hypoxic and inflammatory pulmonary arterial hypertension and smooth muscle proliferation via xanthine oxidoreductase-dependent nitric oxide generation. Circulation. 2010;121:98-109.

13. Alef MJ, Vallabhaneni R, Carchman E, Morris SM Jr, Shiva S, Wang Y, et al. Nitrite-generated NO circumvents dysregulated arginine/NOS signaling to protect against intimal hyperplasia in Sprague-Dawley rats. J Clin Invest. 2011;121: 1646-56.

14. Sugimoto R, Okamoto T, Nakao A, Zhan J, Wang Y, Kohmoto J, et al. Nitrite reduces acute lung injury and improves survival in a rat lung transplantation model. Am J Transplant. September 27, 2012 [Epub ahead of print].

15. Mizuta T, Kawaguchi A, Nakahara K, Kawashima Y. Simplified rat lung transplantation using a cuff technique. J Thorac Cardiovasc Surg. 1989;97:578-81.

16. Kohmoto J, Nakao A, Stolz DB, Kaizu T, Tsung A, Ikeda A, et al. Carbon monoxide protects rat lung transplants from ischemia-reperfusion injury via a mechanism involving p38 MAPK pathway. Am J Transplant. 2007;7:2279-90.

17. Jensen F. The role of nitrite in nitric oxide homeostasis: a comparative perspective. Biochim Biophys Acta. 2009; 1787:841-8.

18. Dezfulian C, Raat N, Shiva S, Gladwin MT. Role of the anion nitrite in ischemia-reperfusion cytoprotection and therapeutics. Cardiovasc Res. 2007; 75:327-38.

19. Duranski MR, Greer JJ, Dejam A, Jaganmohan S, Hogg N, Langston W, et al. Cytoprotective effects of nitrite during in vivo ischemia-reperfusion of the heart and liver. J Clin Invest. 2005;115:1232-40.

20. Tripatara P, Patel N, Webb A, Rathod K, Lecomte F, Mazzon E, et al. Nitrite-derived nitric oxide protects the rat kidney against ischemia/reperfusion injury in vivo: role for xanthine oxidoreductase. J Am Soc Nephrol. 2007; 18:570-80.

21. Kavanagh B, Mouchawar A, Goldsmith J, Pearl R. Effects of inhaled NO and inhibition of endogenous NO synthesis in oxidant-induced acute lung injury. $J$ Appl Physiol. 1994;76:1324-9.

22. Murakami S, Bacha E, Mazmanian G, Détruit H, Chapelier A, Dartevelle P, et al. Effects of various timings and concentrations of inhaled nitric oxide in lung ischemia-reperfusion. The Paris-Sud University Lung Transplantation Group. Am J Respir Crit Care Med. 1997;156:454-8.

23. Dong M, Abano J, Egan T. Nitric oxide ventilation of rat lungs from non-heartbeating donors improves posttransplant function. Am J Transplant. 2009;9: 2707-15.

24. Naka Y, Chowdhury N, Oz M, Smith C, Yano O, Michler R, et al. Nitroglycerin maintains graft vascular homeostasis and enhances preservation in an orthotopic rat lung transplant model. J Thorac Cardiovasc Surg. 1995;109: 206-10.

25. Yamashita M, Schmid R, Ando K, Cooper J, Patterson G. Nitroprusside ameliorates lung allograft reperfusion injury. Ann Thorac Surg. 1996;62:791-6. 


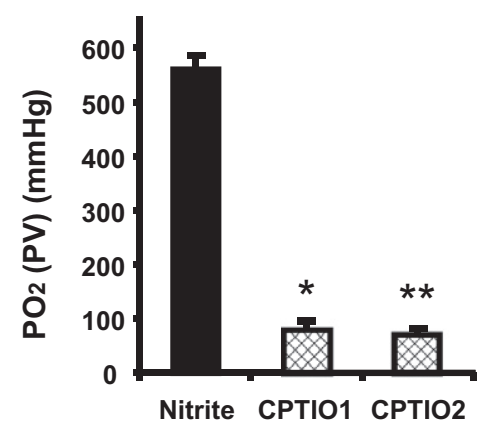

A

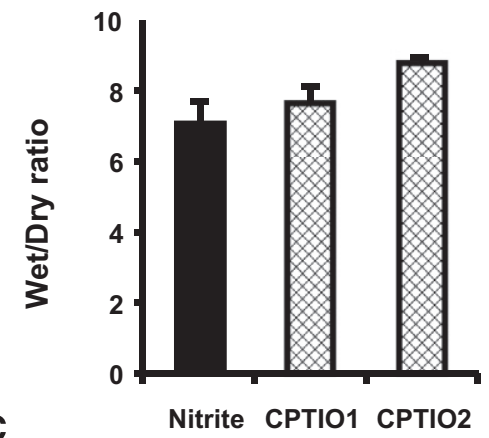

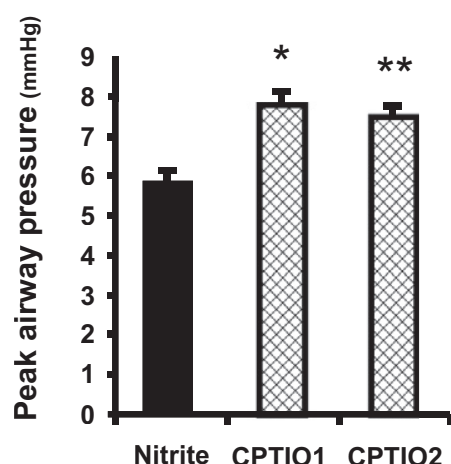

B
Nitrite CPTIO1 CPTIO2

FIGURE E1. Inhibition of nitric oxide $(N O)$ by 2-(4-carboxypheny)-4,4,5,5-tetramethylimidazoline-1-oxyl-3-oxide $(C$-PTIO). A, Pulmonary vein oxygen tension $\left(\mathrm{Po}_{2}\right)$ (inspired oxygen fraction $\left[\mathrm{Fio}_{2}\right]=1.0$ ) was analyzed after 2 hours of reperfusion using 2-(4-carboxypheny)-4,4,5,5-tetramethylimidazoline-1oxyl-3-oxide ( $C$-PTIO). In the CPTIO1 group, where C-PTIO was administered in donor animals before cold ischemia, $\mathrm{PO}_{2}$ was lower than that in the nitrite group $(80.2 \pm 20.3 \mathrm{~mm} \mathrm{Hg}$ vs $560.8 \pm 26.8 \mathrm{~mm} \mathrm{Hg} ; * P<.001)$. In the CPTIO2 group, where C-PTIO was administered in recipient animals $10 \mathrm{minutes}$ before reperfusion, $\mathrm{PO}_{2}$ was lower than that in the nitrite group $(70.8 \pm 12.4 \mathrm{~mm} \mathrm{Hg}$; $* * P<.001)$. B, The peak airway pressure $(P A W P)$ of the trachea was measured after 2 hours of reperfusion. In the CPTIO1 group, PAWP was higher than that in the nitrite group $(7.8 \pm 0.3 \mathrm{~mm} \mathrm{Hg} \mathrm{vs} 5.8 \pm 0.3 \mathrm{~mm} \mathrm{Hg}$; $* P=.006)$. In the CPTIO2 group, PAWP was higher than that in the nitrite group $(7.5 \pm 0.3 \mathrm{~mm} \mathrm{Hg}$ vs $5.8 \pm 0.3 \mathrm{~mm} \mathrm{Hg} ; * * P=.01)$. C, The graft wet/dry ratio was measured after 2 hours of reperfusion. Both the CPTIO1 and CPTIO2 groups had higher wet/dry ratios than the nitrite group $(P=.13) . P V$, Pulmonary vein. 